\title{
ON THE SHAPE OF TORUS-LIKE CONTINUA AND COMPACT CONNECTED TOPOLOGICAL GROUPS ${ }^{1}$
}

\author{
JAMES KEESLING
}

\begin{abstract}
In this paper it is shown that if $X$ is a torus-like continuum, then $X$ has the shape of a compact connected abelian topological group. Let $\Pi$ be a collection of compact connected Lie groups. In light of the above result it is natural to ask if a ПI-like continuum has the shape of a compact connected topological group. An example is given to show that this is not the case.
\end{abstract}

Introduction. Let $C$ denote the category of compact Hausdorff spaces and continuous maps and let $H: C \rightarrow H C$ be the homotopy functor. Let $S: C \rightarrow S C$ be the functor of shape in the sense of Holsztynski for the projection functor $H$ [5]. It is assumed that the reader is familiar with the equivalence of this approach to shape with that of Mardesic and Segal using ANR-systems [12]. A precise statement of this equivalence with a proof is given in the Appendix of [6]. In this paper we give the shape classification of all torus-like continua. If $X$ is a torus-like continuum, then it has the same shape as a compact connected abelian topological group. It is shown, in fact, that $X$ has the same shape as char $H^{1}(X)$ where $H^{n}(X)$ is $n$-dimensional Čech cohomology over the integers. Using our knowledge of the shape properties of compact connected abelian topological groups contained in [6], [7], and [8] several properties of torus-like continua are derived.

In light of the above result about torus-like continua, it is natural to ask if a $\Pi$-like continuum might not have the shape of a compact connected topological group where $\Pi$ is a collection of compact connected Lie groups. An example is given to show that this is not the case. In this section of the paper we also show that if $G$ is a compact connected topological group with $H^{1}(G)=0$, then $H^{n}(G) /$ Tor $H^{n}(G)$ has property L for all $n \geqq 0$. This result is invoked to show that the above example cannot have the shape of a compact connected topological group. An example is

Received by the editors November 20, 1972.

AMS (MOS) subject classifications (1970). Primary 55D99; Secondary $22 \mathrm{~B} 99$.

Key words and phrases. Torus-like continuum, shape, compact connected abelian topological group, compact connected topological group, Cech cohomology, property L, movability.

${ }^{1}$ This research was supported by N.S.F. Grant GP-24616A \#1. 
also given of a nonmovable $G$ with $H^{1}(G)=Z$. In [6] it was shown that for $A$ a compact connected abelian topological group, $A$ is movable if and only if $H^{1}(A)$ has property $\mathrm{L}$. The last example shows that the fact that $A$ is assumed to be abelian is not superfluous in that theorem.

Throughout the paper we let $H^{n}(X)$ denote $n$-dimensional Čech cohomology with integer coefficients. We assume the notation of [6], [7], and $[8]$.

1. The shape of torus-like continua. In this section we show that if $X$ is a torus-like continuum, then $X$ has the shape of a compact connected abelian topological group and we draw some corollaries from this. First we recall the definition of $\Pi$-like.

1.1. Definition. Let $\Pi$ be a collection of polyhedra. Then a continuum $X$ is said to be $\Pi$-like provided that, for every open cover $\mathscr{U}$ of $X$, there is a $P \in \Pi$ and a continuous $f(X)=P$ such that for $p \in P, f^{-1}(p) \subset U$ for some $U \in \mathscr{U}$. If $\Pi$ is the collection of tori, $\Pi=\left\{T^{n}: n \geqq 0\right\}$, then the continuum is said to be torus-like.

1.2. THEOREM. Let $X$ be a torus-like continuum. Then $X$ has the shape of a compact connected abelian topological group.

Proof. First we suppose that $X$ is metrizable. In this case there is an inverse sequence of tori $X=\left\{T_{i}^{n_{i}} ; \pi_{i j} ; i \leqq j<\omega_{0}\right\}$ which has $X$ as inverse limit [10]. Then $X$ is an ANR-sequence associated with $X$ in the terminology of [12]. Let $\pi_{i j}^{*}$ be the unique continuous homomorphism homotopic to $\pi_{i j}$ by [14] and let $A$ be the inverse limit of $A=\left\{T_{i}^{n_{i}} ; \pi_{i j}^{*}\right.$; $\left.i \leqq j<\omega_{0}\right\}$. Then $A$ is a compact connected abelian topological group and $A$ is associated with the ANR-sequence $A$. We define a map of ANR-

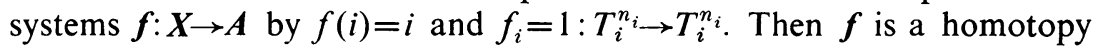
equivalence between the ANR-systems $X$ and $A$. Thus $X$ and $A$ have the same shape (see the Appendix of [6] for the equivalence of the approach to shape in [12] to that in [5]). Now we will proceed to prove the theorem in general. First note that if $X$ and $Y$ are metrizable and torus-like and $F \in \operatorname{Mor}_{S C}\left(X, A_{X}\right)$ and $G \in \operatorname{Mor}_{S C}\left(A_{Y}, Y\right)$ are shape equivalences with $A_{X}$ and $A_{Y}$ compact connected abelian topological groups, then if we define $P: \operatorname{Hom}\left(A_{X}, A_{Y}\right) \rightarrow \operatorname{Mor}_{S C}(X, Y)$ by $P(h)=G \circ S(h) \circ F$, then $P$ is one-to-one and onto by Theorem 1.2 of [6].

Suppose now that $X$ is a torus-like continuum which is not metrizable. Then by [9], there is an inverse system $\left\{X_{\alpha} ; \pi_{\alpha \beta} ; \alpha \leqq \beta \in A\right\}$ having $X$ as its inverse limit such that each $X_{\alpha}$ is metrizable and torus-like. For each $\alpha \in A$, let $A_{\alpha}$ be a compact connected abelian topological group and $F_{\alpha} \in \operatorname{Mor}_{S C}\left(X_{\alpha}, A_{\alpha}\right)$ and $G_{\alpha} \in \operatorname{Mor}_{S C}\left(A_{\alpha}, X_{\alpha}\right)$ such that $F_{\alpha} \circ G_{\alpha}$ is the identity shape morphism on $A_{\alpha}$ and $G_{\alpha} \circ F_{\alpha}$ is the identity shape morphism 
on $X_{\alpha}$. Then for each $\alpha \leqq \beta$, let $\pi_{\alpha \beta}^{*}$ be the unique continuous homomorphism from $A_{\beta}$ to $A_{\alpha}$ such that $S\left(\pi_{\alpha \beta}\right)=G_{\beta} \circ S\left(\pi_{\alpha \beta}^{*}\right) \circ F_{\alpha}$ as above. Then $\left\{A_{\alpha} ; \pi_{\alpha \beta}^{*} ; \alpha \leqq \beta \in A\right\}$ becomes an inverse system of compact connected abelian topological groups. Let $A$ be the inverse limit of this inverse system. Then $A$ is a compact connected abelian topological group. It now follows easily from the continuity of the shape functor [5] that $S(A)=S(X)$ and the proof is complete.

Using the results of [6], [7], and [8] we now give some immediate consequences of Theorem 1.2.

1.3. Corollary. If $X$ is a torus-like continuum, then $X$ has the same shape as char $H^{1}(X)$.

Proof. By Theorem 1.2 there is a compact connected abelian topological group $A$ which is shape equivalent to $X$. This implies that $H^{1}(X) \simeq$ $H^{1}(A)$. Let $A_{X}=$ char $H^{1}(X)$. Then $H^{1}(X) \simeq H^{1}\left(A_{X}\right)$ by Corollary 1.5 of [8]. Thus $H^{1}(A) \simeq H^{1}\left(A_{X}\right)$. By Theorem 1.4 of [8], char $A \simeq \operatorname{char} A_{X}$. This implies that $A \simeq A_{X}$ and that $X, A$, and $A_{X}$ all have the same shape.

1.4. Corollary. Let $X$ be a continuum and $Y$ a torus-like continuum. Then $\operatorname{Mor}_{S C}(X, Y)$ is in one-to-one correspondence with $\operatorname{Hom}\left(H^{1}(Y), H^{1}(X)\right)$ by the function which takes $F \in \operatorname{Mor}_{S C}(X, Y)$ to the homomorphism $F^{*}$.

Proof. By Theorem 1.7 of [8] this is true for $Y$ a compact connected abelian topological group and since $Y$ has the same shape as a compact connected abelian topological group, the corollary follows.

1.5. Corollary. Let $X$ be a torus-like continuum. Then $X$ is movable if and only if $H^{1}(X)$ has property $\mathrm{L}$.

Proof. This is because this is true for compact connected abelian topological groups by Theorem 2.5 of [6] and Theorem 1.4 of [8].

Of course, the Čech cohomology of a torus-like continuum $Y$ will be the same as its associated compact connected abelian topological group. Thus one can apply the results of [3] to compute the Cech cohomology of $Y$ over any integral domain $R$.

In the next section we will show that if $\Pi$ is a collection of compact connected Lie groups, then a continuum $X$ may be $\Pi$-like without having the shape of a compact connected topological group.

2. Compact connected topological groups. In this section we use a well-known structure theorem for compact connected topological groups to derive a shape invariant property for such spaces. This is used to show that a certain continuum cannot have the shape of a compact connected topological group. 
2.1. THEOREM. Let $G$ be a compact connected topological group. Then $G$ is isomorphic to a group $(A \times B) / D$ where $A$ is a product of simple, connected, simply connected compact Lie groups; $B$ is a compact connected abelian topological group; and $D$ is a totally disconnected closed central subgroup of $A \times B$.

This is essentially 6.59 on p. 75 in [2]. Now we recall the definition of property L.

2.2. Definition. Let $H$ be an abelian group and $J$ a subgroup of $G$. Then $J$ is said to admit division if whenever $h \in H$ and $n$ is a positive integer, then $n h \in J$ implies that $h \in J$. This is equivalent to saying that $H / J$ is torsion free. The group $H$ is said to have property $\mathrm{L}$ if every finite subset of $H$ is contained in a finitely generated subgroup that admits division.

In [8] it was shown that if $X$ is a movable continuum, then $H^{n}(X) /$ Tor $H^{n}(X)$ has property $\mathrm{L}$ for all $n \geqq 0$. This fact will be used in the proof of the next theorem. We also note that if $X=\prod_{\alpha \in A} X_{\alpha}$ where each $X_{\alpha}$ is an ANR, then $X$ is movable. This can be seen by letting $X$ be the limit of the finite subproducts $\prod_{i=1}^{n} X_{\alpha_{i}}$ with the bonding maps just projections onto subproducts. This is clearly a movable ANR-system associated with $X$.

2.3. THEOREM. Let $G$ be a compact connected topological group. Then if $H^{1}(G)=0$, then $H^{n}(G) /$ Tor $H^{n}(G)$ has property $\mathrm{L}$ for all $n \geqq 0$.

Proof. Let $G=(A \times B) / D$ as in Theorem 2.1 and suppose that $H^{1}(G)=$ 0 . Then suppose that $B \neq 0$. Then let $\pi_{B}: A \times B \rightarrow B$ be the projection map. Now $\pi_{B}$ is a continuous homomorphism. Therefore $\pi_{B} \mid D$ is a continuous homomorphism and thus $\pi_{B} \mid D: D \rightarrow \pi_{B}(D)$ is open and thus $\pi_{B}(D)$ is also 0 -dimensional since $\pi_{B} \mid D$ is open and closed. This implies that $B / \pi_{B}(D) \neq 0$. Let $H$ be the commutator subgroup of $G$, then $G / H \simeq$ $B / \pi_{B}(D) \neq 0$. By Theorem 3.1 of $[8], H^{1}(G / H) \simeq H^{1}(G) \neq 0$, a contradiction. That is, if $H^{1}(G)=0$, then $B=0$. Thus $G \simeq A / D$. Now $A=\prod_{\gamma \in \Gamma} L_{\gamma}$ where each $L_{\gamma}$ is a simple, simply connected, compact connected Lie group. Since each $L_{\gamma}$ is an ANR, $A$ is movable. Thus $H^{n}(A) /$ Tor $H^{n}(A)$ has property $\mathrm{L}$ for all $n \geqq 0$ [8, Theorem 4.4]. By [4, 3.16, p. 330], the quotient homomorphism $p: A \rightarrow A / D=G$ induces an injection $p^{*}: H^{n}(G) /$ Tor $H^{n}(G) \rightarrow H^{n}(A) /$ Tor $H^{n}(A)$. Now a subgroup of a group having property $\mathrm{L}$ has property $\mathrm{L}$. Thus $H^{n}(G) /$ Tor $H^{n}(G)$ has property L for all $n \geqq 0$.

2.4. EXAMPLE. Here we will show that if $X$ is a $\Pi$-like continuum where $\Pi$ is a collection of compact connected Lie groups, then $X$ need not have the shape of a compact connected topological group. Let $\Sigma_{a}$ be a solenoid 
which is not a circle. Then $H^{1}\left(\Sigma_{a}\right)=$ char $\Sigma_{a}$ does not have property $\mathrm{L}$ and is torsion free. Let $X=\Sigma^{2} \Sigma_{a}$ be the two-fold suspension of $\Sigma_{a}$. Then $X$ is a 3-sphere-like continuum with the 3-sphere a compact connected Lie group. However, $H^{3}(X) \simeq H^{1}\left(\Sigma_{a}\right)$ is torsion free and does not have property L. Also, $H^{1}(X)=0$. Thus $X$ cannot have the shape of a compact connected topological group.

In [6] it was shown that if $A$ is a compact connected abelian topological group, then $A$ is movable if and only if $H^{1}(A)$ has property L. We will now give an example of a compact connected topological group $G$ which is movable with $H^{1}(G)=Z$. This shows that this theorem is not true for compact connected topological groups.

2.5. EXAMPLE. Recall that the center of $S U(k)=L_{k}$ is just the cyclic group $Z_{k}\left[1\right.$, p. 31]. Let $\left\{p_{i}\right\}$ be a sequence of increasing prime numbers. Observe that one can imbed $Z_{p_{1} \cdots p_{n}} \subset Z_{p_{1}} \times \cdots \times Z_{p_{n}} \subset L_{p_{1}} \times \cdots \times L_{p_{n}}$ by the homomorphism $1 \mapsto(1,1, \cdots, 1)$. One can also imbed $Z_{p_{1} \cdots p_{n}}$ in the circle group $T$ as the $\left(p_{1} \cdots p_{n}\right)$ th roots of unity. Consider $Z_{p_{1} \cdots p_{n}} \times$ $Z_{p_{1} \cdots p_{n}} \subset T \times\left[L_{p_{1}} \times \cdots \times L_{p_{n}}\right]$ as the product of these two imbeddings. Then consider $\Delta: Z_{p_{1} \cdots p_{n}} \rightarrow Z_{p_{1} \cdots p_{n}} \times Z_{p_{1} \cdots p_{n}}$ as the diagonal map and let $D_{n}=\Delta\left(Z_{p_{1} \cdots p_{n}}\right) \subset Z_{p_{1} \cdots p_{n}} \times Z_{p_{1} \cdots p_{n}} \subset T \times\left[L_{p_{1}} \times \cdots \times L_{p_{n}}\right]$. Let $G_{n}=$ $\left[T \times\left(L_{p_{1}} \times \cdots \times L_{p_{n}}\right)\right] / D_{n}$. Let $\quad \psi_{n m}: T \times\left(L_{p_{1}} \times \cdots \times L_{p_{n}}\right) \rightarrow T \times\left(L_{p_{1}} \times\right.$ $\left.\cdots \times L_{p_{n}}\right)$ be defined by $\psi_{n m} \mid T$ is equivalent to the complex map $z \mapsto z^{p_{n+1} \cdots p_{m}}$ and $\psi_{n m}\left(L_{p_{1}} \times \cdots \times L_{p_{m}}\right)=L_{p_{1}} \times \cdots \times L_{p_{n}}$ is just the projection onto the first $n$ factors. Note that $\psi_{n m}\left(D_{m}\right)=D_{n}$. Thus $\psi_{n m}$ induces a continuous homomorphism $\pi_{n m}: G_{m} \rightarrow G_{n}$. Clearly $\pi_{n m} \circ \pi_{m p}=$ $\pi_{n p}$ for $n \leqq m \leqq p$. Thus we have an inverse sequence of compact connected Lie groups $\left\{G_{n} ; \pi_{n m} ; n \leqq m<\omega_{0}\right\}$. Let $G$ be the inverse limit group. Then we claim that $H^{1}(G)=Z$ and that $G$ is nonmovable.

First we show that $G$ is nonmovable. This is equivalent to showing that the ANR-sequence $\left\{G_{n} ; \pi_{n m} ; n \leqq m<\omega_{0}\right\}$ is not movable. Note that the fundamental group of $G_{n}$ is $\pi_{1}\left(G_{n}\right)=Z \times Z_{p_{1} \ldots p_{n}}$ and $\pi_{n m}^{*}: \pi_{1}\left(G_{m}\right) \rightarrow$ $\pi_{1}\left(G_{n}\right)$ is the homomorphism taking $(1,0)$ to $\left(p_{n+1} \cdots p_{m}, 0\right)$ and $(0,1)$ to $(0,1)$. Let $n=1$. Then in the definition of movability one must be able to find an $m \geqq 1$ such that for all $p \geqq m$, there is a continuous map $r^{m p}$ : $G_{m} \rightarrow G_{p}$ such that $\pi_{1 m}$ is homotopic to $\pi_{1 p} \circ r^{m p}$. However, this would insure that there is a homomorphism $h^{m p}: \pi_{1}\left(G_{m}\right) \rightarrow \pi_{1}\left(G_{p}\right)$ such that $\pi_{1 m}^{*}=\pi_{1 p}^{*} \circ h^{m p}$. However, letting $p=m+1$, no such $h^{m p}$ can exist. Thus $G$ cannot be movable.

To show that $H^{1}(G)=Z$ note that if $Q$ is the commutator subgroup of $G$, then the quotient homomorphism $p: G \rightarrow G / Q$ induces an isomorphism $p^{*}: H^{1}(G / Q) \simeq H^{1}(G)$ by Theorem 3.1 of [8]. But $G / Q$ is the inverse limit of $G_{n} / Q_{n}$ where $Q_{n}$ is the commutator subgroup of $G_{n}$. Now $Q_{n}=$ $L_{p_{1}} \times \cdots \times L_{p_{n}}$ and $G_{n} / Q_{n} \simeq T$ for all $n$. Now the induced homomorphism 
from $G_{m} / Q_{m} \rightarrow G_{n} / Q_{n}$ is an isomorphism. Thus the inverse limit of $G_{n} / Q_{n}$ is just $T$ and $G / Q \simeq T$. Thus $H^{1}(G / Q) \simeq H^{1}(G) \simeq Z$.

\section{REFERENCES}

1. G. Bredon, Introduction to compact transformation groups, Academic Press, New York, 1972.

2. K. H. Hofmann, Introduction to the theory of compact groups, Part II, Tulane University Lecture Notes, Tulane University, 1968.

3. Categories with convergence, exponential functors, and the cohomology of compact abelian groups, Math. Z. 104 (1968), 106-140. MR 37 \#4195.

4. K. H. Hofmann and P. S. Mostert, Elements of compact semigroups, Charles E. Merrill Books, Columbus, Ohio, 1966. MR 35 \#285.

5. W. Holsztyński, An extension and axiomatic characterization of Borsuk's theory of shape, Fund. Math. 70 (1971), no. 2, 157-168. MR 43 \#8080.

6. J. Keesling, Shape theory and compact connected abelian topological groups (submitted).

7. - Continuous functions induced by shape morphisms, Proc. Amer. Math. Soc. (to appear).

8. - An algebraic property of the Čech cohomology groups which prevents local connectivity and movability (submitted).

9. S. Mardesič, e-mappings and inverse limits, Glasnik Mat.-Fiz. Astronom. Društvo Mat. Fiz. Hrvatski Ser. II. 18 (1963), 195-205. MR 29 \#2783.

10. S. Mardesič and J. Segal, e-mappings and polyhedra, Trans. Amer. Math. Soc. 109 (1963), 146-164. MR 28 \#1592.

11. - Movable compacta and ANR-systems, Bull. Polon. Acad. Sci. Sér. Sci. Math. Astronom. Phys. 18 (1970), 649-654. MR 44 \#1026.

12. - Shapes of compacta and ANR-systems, Fund. Math. 72 (1971), 41-59.

13. L. S. Pontrjagin, Continuous groups, 2nd ed., GITTL, Moscow, 1954; English transl., Gordon and Breach, New York, 1966. MR 17, 171; MR 34 \#1439.

14. W. Scheffer, Maps between topological groups that are homotopic to homomorphisms, Proc. Amer. Math. Soc. 33 (1972), 562-567.

Department of Mathematics, University of Florida, Gainesville, Florida 32601 